\title{
Editorial
}

\section{Anticipating the Household Robots}

As we are immersed in the hand-held devices, perhaps it is time now to think of another big event-the the arrival of automation and robots. It may seem very distant or vague at present but nobody can predict the arrival of some epoch-making technology. May be there would be a convergence of the so-called smart phones and household robots. Robots are there, of course, in many fields, but here we are talking about the arrival of robots for serving our household needs and personal assistance; for example, robots helping people with cooking, washing, cleaning and other repetitive activities. Even, by using such robots we can do away with certain services, which sometimes demand lowering of human dignity.

However, our attitude to robots differs from smart phones in one important aspect: our distrust of robots at home. There are several reasons behind such attitude, and here we will talk about only one aspect: design. Much of our aversion to robots lie with the stereotypical designs of robots made popular or notorious through a sci-fi presentation of the devices. In an effort to project a kind of machine-human combination, robots have been perceived as having something to do with the alien. This tendency might have emerged in the west because of polarity in the thinking pattern, whose roots are to be traced back to the duality of God and Devil. Just as anything bad was associated with the Devil, the probable sinister aspect of the robots was thought in line with that kind of Christian theological paradigm. This needs a shift in design conception.

In all other sphere we think of the design of the devices as per the demand of the components and ergonomics. The same needs to be hammered home while understanding robots and while designing robots. Household robots will be smaller and even tiny in sizes and design must follow utility and aesthetics. Industrial aesthetics depend heavily economy and in many cases, the economy dictates aesthetics. But there are counter-forces too. Such forces may not be perceptible at once or may not challenge the economy instantly. Rather such forces remain hidden and operate in subversive manner. An example of such force can be found with folk art forms, particularly the performance arts which pose a resistance to acquisition of aesthetics by economy. So the designers need to understand that attempt at achieving global uniformity may not go well with all the sections.

The market economy cannot conquer or appropriate everything. Our situation demands a truce in order to satisfy the sensibility of the customers. What we are basically suggesting is that production of household robots will need naturalization in a particular culture and the naturalization process will demand designing in consonance with the fundamental aesthetics of a particular culture. Let us wait for day. 